Leukemia - Lymphoma and Myeloma

\title{
Follicular Lymphoma in Young Adults: Study from a Regional Cancer Center in South India
}
A. H. Rudreshaa ${ }^{1}$ Shina Goyal ${ }^{1}$
D. Lokanatha ${ }^{1}$ Linu Abraham Jacob ${ }^{1,1}$
M. C. Suresh Babu'
K. N. Lokesh ${ }^{1}$ Smitha Saldanha ${ }^{1}$
Bipinesh Sansar ${ }^{1}$
L. K. Rajeev ${ }^{1}$

${ }^{1}$ Department of Medical Oncology, Kidwai Memorial Institute of

Address for correspondence Shina Goyal, MD, DM (Medical Oncology), Department of Medical Oncology, Kidwai Memorial Institute of Oncology, Bengaluru, Karnataka, India (e-mail: shina. goyal@gmail.com).

\section{South Asian J Cancer 2021;10:115-119.}

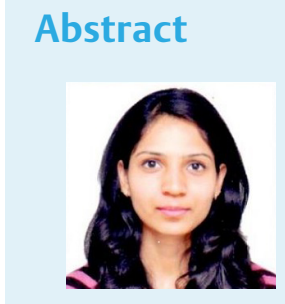

Shina Goyal

Keywords

- follicular lymphoma

- outcomes

- young adults
Objective Follicular lymphoma (FL) is a disease of the elderly. It is postulated that younger patients have distinct tumor biology and treatment outcomes. Various lymphoma groups across the world have studied this to understand if young adults (YAs) need a different treatment approach. Our study fills the void in data from an Asian country on YA population with FL.

Patients and Methods We retrospectively analyzed young patients (age $\leq 40$ years) diagnosed with FL at our center from 2012 to 2018. Their disease characteristics, treatment details, and outcomes were studied to examine any association between various parameters and survival.

Results Therewere 28 young FL patients included in our study that constituted $14.6 \%$ of FL cases (males: $53.5 \%$ and females: $46.5 \%$ ). The median age at diagnosis was 36.5 years. Most of the patients presented in an advanced stage, $57 \%$ had extranodal involvement, and $39.3 \%$ had bone marrow involvement at the time of presentation. The most common chemotherapy regimen used was cyclophosphamide, vincristine, and prednisone. Half of them received chemoimmunotherapy and only $18 \%$ continued rituximab as maintenance therapy. The overall response rate was $92.9 \%(n=26)$, and the remaining two patients had progressive disease while on treatment. The median progression free survival (PFS) was 6.1 years and median overall survival (OS) was not reached. On univariate analysis, extranodal disease was associated with a lower PFS $(p=0.06)$ and low hemoglobin showed a significant association with OS $(p=0.005)$. On multivariate analysis, none of the factors showed a significant association with survival.

Conclusion Most YAs present with advanced disease with a good response to treatment and favorable outcomes.
How to cite this article: Rudresha H. A, Goyal S, Lokanatha D, Jacob A. L, Lokesh N. K, Saldanha S, Sansar B, Rajeev K. L. 2278330X.Original ArticleFollicular Lymphoma in Young Adults: A Study from a Regional Cancer Center in South India. South Asian J Cancer 2021;10(2):115-119.
(C) 2021. MedIntel Services Pvt Ltd.

MedIntel Services Pvt Ltd.. This is an open access article published by Thieme under the terms of the Creative Commons Attribution-NonDerivative-NonCommercial-License, permitting copying and reproduction so long as the original work is given appropriate credit. Contents may not be used for commercial purposes, or adapted, remixed, transformed or built upon. (https://creativecommons.org/licenses/by-nc-nd/4.0/). Thieme Medical and Scientific Publishers Private Ltd A-12, Second Floor, Sector -2, NOIDA -201301, India 


\section{Introduction}

Follicular lymphoma (FL) is the second most common subtype of non-Hodgkin's lymphoma (NHL) (20\% of NHL) and the most common of the clinically indolent lymphomas. ${ }^{1}$ Despite the recent developments and improvements in survival, FL remains an incurable disease. The median survival is 10 to 14 years with disease progression in $20 \%$ at 2 years including a transformation rate of $10 \%$ at 5 years. ${ }^{2}$

FL is a disease of the elderly with the median age at diagnosis of 67 years and a slight female predominance. ${ }^{3}$ In a report of the National LymphoCare Study (NLS) by Nabhan et al, they found that among Caucasians, those aged $<45$ years accounted for $9.3 \%$ of patients with $\mathrm{FL}$ and higher rates were noted in African-American (25.3\%) and Hispanic populations (22.4\%). ${ }^{4}$ We have vast data on the natural course, prognosis, and outcomes of this indolent lymphoma in the older age group. Young adult (YA) population is under-represented in clinical trials due to its rarity, and hence, we lack understanding of its biologic behavior in this population. Considering longer survival in indolent lymphomas, they may have distinct treatment outcomes, long-term toxicity, higher transformation rates, and distinct psychosocial consequences from a cancer diagnosis.

This prompted us to study this subgroup of patients at our institute to understand their clinicopathological characteristics, treatment outcomes, and prognostic factors.

\section{Patients and Methods}

The clinical information about patients diagnosed with FL between January 2012 and June 2018 at Kidwai Cancer Institute, Bengaluru, Karnataka, was retrospectively collected from the record files. Those between 18 and 40 years of age with newly diagnosed FL were classified as YA patients and were included for analysis. These records were further reviewed for information on demographics (age, gender, and date of diagnosis) and clinicopathological features (histological grade, nodal and extranodal involved sites, stage, B symptoms, and serum lactate dehydrogenase [LDH]), first-line treatment, time of progression, and outcome (duration of survival). The FL International Prognostic Index (FLIPI) score $^{5}$ was calculated by using the information. The Groupe d'Etude des Lymphomes Folliculaires (GELF) ${ }^{6}$ and the British National Lymphoma Investigation ${ }^{7}$ criteria were used to determine the disease extent and for defining management strategies. FL is graded based on the proportion of centrocytes to centroblasts. Grade 3 is further subdivided into $3 \mathrm{~A}$ and $3 \mathrm{~B}$, of which grade $3 \mathrm{~B}$ is a biologically distinct entity and behaves like a high-grade lymphoma. ${ }^{8}$ Thus, patients with grade 3B disease were excluded from the study.

Response criteria for malignant lymphoma were used to determine the response to chemotherapy. Imaging used in our study was a contrast computed tomography (CT) scan except for two patients who underwent positron emission tomography-CT (PET-CT) for response assessment. Progression-free survival was defined as the time from date of diagnosis to the date of occurrence of progressive disease.
Progression-free survival at 24 months (PFS24) was defined for patients with a minimum 2-year follow-up. Overall survival (OS) was calculated from the date of diagnosis to death or day of last follow-up.

\section{Statistical Analysis}

Survival curves were estimated by using the Kaplan-Meier method. The logistic regression and Cox proportional hazard model were used for univariate and multivariate analyses. The statistical analysis was conducted by using SPSS software v15.

\section{Results}

\section{Patient Characteristics}

We identified 191 patients of FL during the specified period, out of which 28 (14.6\%) patients were YAs. The median age for the YA group was 36.5 years (range: $18-40$ years), out of which $52.2 \%(n=15)$ were men and $47.8 \%(n=13)$ were women.

Most of our patients presented with primary nodal disease, and only two patients (7.1\%) had a primary extranodal disease (ovarian mass and paravertebral mass). The most involved nodal anatomic site was the cervical lymph nodes, followed by the inguinal lymph nodes. Extranodal involvement was present in $57.1 \%$ of patients and bone marrow (BM) involvement in 39.3\% $(n=11)$ of our patients. As per FLIPI scoring, 13 patients (46.4\%) belonged to the high-risk group, while 10 (35.7\%) and 5 (18\%) patients belonged to intermediate- and low-risk groups, respectively. Ninety percent of YA patients seen at our center presented with advanced stage. Only one patient was kept on observation, as there was no indication of treatment as per GELF criteria. However, the patient progressed within 4 months requiring chemotherapy, following which she achieved CR and was disease free. The remaining patients received upfront treatment, and the most used chemotherapy regimen was cyclophosphamide, vincristine, and prednisone with or without rituximab. Half of our patients received rituximab, out of which 5 (17.8\%) patients continued it as maintenance therapy. - Table 1 summarizes other disease characteristics and treatment regimens.

\section{Outcomes}

The overall response rate (ORR) and complete remission (CR) were $92.9 \%(n=26)$ and $39.3 \%(n=11)$, respectively. Two patients had progressive disease while on treatment. The median PFS was 6 years (95\% confidence interval: 1.4-10.6) and median OS was not reached. The 2-year and 5-year PFS was 62.5 and $51 \%$, while the 2 -year and 5 -year OS was 96.2 and $86.1 \%$, respectively. Based on the FLIPI scores, the 2-year PFS for low-, intermediate-, and high-risk groups was 80.1, 66.7, and $52.8 \%$, respectively ( - Fig. 1 ).

On univariate analysis, low hemoglobin ( $\mathrm{Hb}<12 \mathrm{mg} / \mathrm{dL})$ and extranodal disease showed a trend toward lower PFS that did not reach statistical significance. For OS, low hemoglobin was significantly associated with inferior outcomes $(p=$ 0.002 ), while the other factors had no statistically significant 
Table 1 Clinicopathological features and treatment regimens

\begin{tabular}{|c|c|}
\hline Feature & $n(\%)$ \\
\hline \multicolumn{2}{|l|}{ Gender } \\
\hline Male & $15(52.2)$ \\
\hline Female & $13(47.8)$ \\
\hline ECOG PS $0 / 1$ & 25 (86.9) \\
\hline BM involvement & $11(39.3)$ \\
\hline \multicolumn{2}{|l|}{ Stage } \\
\hline I and || & $2(7.2)$ \\
\hline III & $10(35.7)$ \\
\hline IV & $16(57.1)$ \\
\hline Nodal sites $>4$ & $18(64.3)$ \\
\hline $\mathrm{Hb}<12$ & $10(35.7)$ \\
\hline $\mathrm{LDH}>\mathrm{ULN}$ & $12(42.9)$ \\
\hline \multicolumn{2}{|l|}{ FLIPI } \\
\hline Low & $5(17.9)$ \\
\hline Intermediate & $10(35.7)$ \\
\hline High & $13(46.4)$ \\
\hline \multicolumn{2}{|l|}{ Histological grade } \\
\hline 1 & $10(35.7)$ \\
\hline 2 & $12(42.9)$ \\
\hline $3 \mathrm{~A}$ & $6(21.4)$ \\
\hline BCL 2 positive & $22(78.5)$ \\
\hline \multicolumn{2}{|l|}{ Chemotherapy regimen } \\
\hline CVP & $9(32.2)$ \\
\hline $\mathrm{CHOP}$ & $3(10.7)$ \\
\hline Bendamustine (single agent) & $2(7.1)$ \\
\hline RCVP & $5(17.9)$ \\
\hline RCHOP & $4(14.3)$ \\
\hline BR & $5(17.9)$ \\
\hline Extranodal involvement & $16(57.1)$ \\
\hline
\end{tabular}

Abbreviations: $\mathrm{BCL}$, B cell lymphoma; BM, bone marrow; BR, bendamustine and rituximab; CHOP, cyclophosphamide, doxorubicin, vincristine, and prednisone; CVP, cyclophosphamide, vincristine, and prednisone; ECOG PS, Eastern Cooperative Oncology Group performance status; FLIPI, Follicular Lymphoma International Prognostic Index; Hb, hemoglobin; LDH, lactate dehydrogenase; RCHOP, rituximab CHOP; RCVP, rituximab CVP; ULN, upper limit of normal.

influence on OS. The use of rituximab-based regimens and maintenance rituximab showed a trend toward improved PFS and OS. The presence of a complete response after chemotherapy was associated with better OS (5-year OS of 100 vs. $76 \%, p=0.06$ ). On multivariate analysis, none of the studied prognostic factors showed a significant association with survival outcomes.

There were 21 patients included for the PFS24 analysis, and it was found that those who had progression-free survival of over 2 years had a significantly longer OS compared with those who progressed within 2 years (6-year OS of 85.6 vs. $62.3 \%, p=0.04$ ).

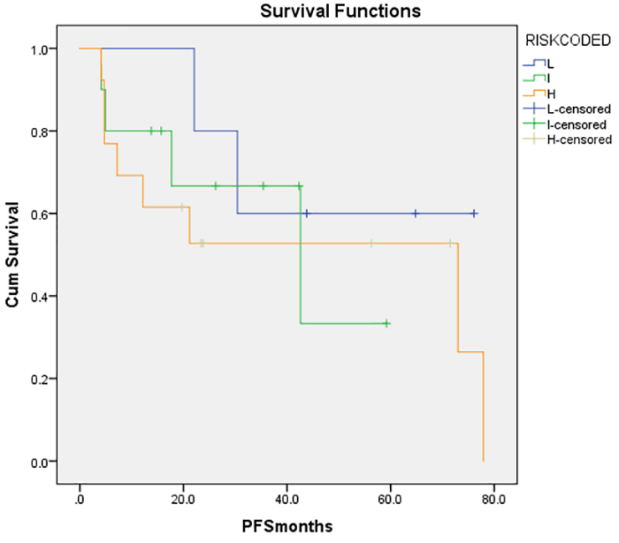

Fig. 1 Kaplan-Meier curve showing progression free survival based on Follicular Lymphoma International Prognostic Index risk scoring.

On follow-up, one patient had disease transformation to grade $3 \mathrm{~B} \mathrm{FL}$ and one patient had disease transformation to DLBCL.

\section{Discussion}

Age is a well-known prognostic factor in FL, and presentation before the age of 40 years is rare. There are very few published studies specifically addressing the characteristics and outcomes of FL in younger adults with no data from India. The disease-specific outcomes and complex psychosocial needs of YAs with malignancies have always attracted increased attention, especially with incurable conditions, such as FL. There may be social factors including interrupted education and loss of work productivity with associated financial concerns. As YAs have a long life expectancy, they may face long-term treatment complications including the risk of second malignancy and transformations, which we need to keep in mind while discussing and planning treatment for this subgroup.

In the present series, the patients younger than 40 years represented $14.6 \%$ of FL cases at our center, and this proportion was higher than that reported from NLS and Surveillance, Epidemiology, and End Results (SEER) database (6 and 5\%, respectively) but similar to data reported from Patient's Medical Home, Canada (13.4\%), and series reported from four European oncology centers (15.4\%). ${ }^{9-11}$ Most of the patients at our center presented with advanced disease. In total, $57 \%$ had extranodal involvement and 39\% showed BM involvement. This was similar to data from one of the largest cohorts of 2,652 FL patients reported from NLS by Casulo et al, ${ }^{11}$ in which 6\% were YAs. Among YAs, 69\% had advanced disease, $57 \%$ had extranodal involvement, and a slightly higher percentage of patients (48\%) had BM involvement.

In our patient population, over 90\% presented with advanced stage and significant symptoms requiring upfront treatment. This could represent the lack of awareness in a developing country like ours, where patients seek medical help only when they develop significant symptoms interfering with their daily work. This is in contrast to a western 
population-based observational study on 46 YAs from the north of England and Scotland ${ }^{12}$ that reported stage III to IV FL in only $74 \%$ of patients and $15 \%$ of them could be kept on observation.

FLIPI was developed in the prerituximab era and predicts disease behavior and survival. It includes the following parameters: age, hemoglobin (patient factors), Ann Arbor stage, number of nodal sites, and serum LDH level (disease factors). Using this index, three risk groups of roughly the same size have been defined. In the low-risk group ( $0-1$ adverse factor), the 5 -year OS is $90 \%$ and in intermediate- and high-risk groups is 77 and $52 \%$, respectively. ${ }^{5}$ Unlike the western literature that has more patients in low-risk groups, in our series, we found a higher percentage of patients with lower hemoglobin, elevated LDH, and high number of nodal sites, and thus, almost half of our patients had high-risk disease according to FLIPI.

Among YAs, Conconi et $\mathrm{al}^{10}$ reported 2-year PFS of $85 \%$ and 5 -year PFS of $60 \%$, while NLS data showed PFS at 2 and 5 years of 75 and $62 \%$. A larger proportion of our patients presented with high-risk FLIPI scores that could explain the inferior outcomes noted in our series.

Attainment of complete response was associated with improved survival in our study. Although PET-CT is a better modality to assess response-in a resource-limited setting like ours-except for two patients who underwent PET-CT, we assessed our patients using a CT scan. Trotman et al have proven in a multicentric analysis that response to chemoimmunotherapy is important and patients with a positive PET scan have a lower progression-free rates at 4 years and inferior OS compared with those who had a negative PET scan ( 23 vs. $63 \%, p<0.0001,4$-year OS, 87.2 vs. $97.1 \%$ ).

Casulo et al looked into a new endpoint to predict outcomes in FL. They analyzed patients from NLCS who received the RCHOP regimen and found that the progression of disease (POD) within 2 years after diagnosis was associated with poor outcomes and high risk for death. ${ }^{13}$ Five-year OS was lower in the early POD group than in the reference group (50 vs. $90 \%$ ). They concluded that it should be further validated as a standard endpoint for chemoimmunotherapy trials. Maurer et $\mathrm{a}^{14}$ have studied this outcome for DLBCL in patients who received chemoimmunotherapy and found that patients alive without progression at 24 months have excellent outcomes. This approach requires further multicentric studies for validation. We attempted to study this newer entity of PFS24 among our patients and found improved survival in patients who were progression free at 2 years.

There is a lack of Indian studies reporting outcomes of FL patients. Gogia et a ${ }^{15}$ reported outcomes of 181 patients of FL of age 24 to 80 years, and the median OS and EFS were 5.5 and 2.5 years, respectively, with a median follow-up of 3 years. ORR and CR were 70 and 35\%, respectively. They reported lower survival outcomes than western data, a difference that is seen in our study as well. Other than factors such as a later stage at presentation and lesser use of rituximab, they postulated the possibility of different biology. We need more prospective data from our country on FL with special emphasis on YA population.
YApatients with cancer face specific challenges. Gupta etal ${ }^{16}$ took an important step in this direction by assessing the supportive care preferences and needs of these YAs with cancer. Our understanding of the needs of YAs with FL, and other indolent hematological malignancies is incomplete. The Spanish Lymphoma Oncology Group ${ }^{17}$ series on YA patients of FL observed longer survival in those who were younger than 40 years at diagnosis $(p<0.00001)$ and proposed that 40 years should be considered as the cutoff age in the prognostic evaluation of patients with FL rather than the classic 60 years of age. All studies are in concordance with the above results of better survival outcomes for YAs. The present evidence does not recommend any differences in treatment in this younger population. However, given that survivorship in this patient population is not synonymous with a cure, we need to further study the tumor biology and outcomes with an emphasis on long-term toxicity that may call for a different treatment approach for YA patients.

The retrospective nature of the study, small sample size, and short period of follow-up form the limitations of our series. The chemotherapy regimens used varied among patients with less than half of the patients receiving rituximab.

In our study, we found that YAs constituted a larger proportion of FL patients compared with our western counterparts. More often, they presented with advanced stage and required upfront treatment. They survive longer with the disease. The survival rates seen are comparable to another Indian study but lower than the western data.

\section{Funding}

None.

\section{Conflict of Interest}

None declared.

\section{Acknowledgment}

The authors thank the support of medical record department staff in helping to retrieve the patients' data.

\section{References}

1 A clinical evaluation of the International Lymphoma Study Group classification of non-Hodgkin's lymphoma. The NonHodgkin's Lymphoma Classification Project. Blood 1997; 89(11):3909-3918

2 Link BK, Maurer MJ, Nowakowski GS, et al. Rates and outcomes of follicular lymphoma transformation in the immunochemotherapy era: a report from the University of Iowa/MayoClinic Specialized Program of Research Excellence Molecular Epidemiology Resource. J Clin Oncol 2013;31(26):3272-3278

3 Schmidt C, Fetscher S, Görg C, et al. Treatment of indolent lymphoma in Germany - results of a representative population-based survey. Clin Lymphoma Myeloma Leuk 2011;11(2): 204-211

4 Nabhan C, Byrtek M, Taylor MD, et al. Racial differences in presentation and management of follicular non-Hodgkin lymphoma in the United States: report from the National LymphoCare Study. Cancer 2012;118(19):4842-4850

5 Solal-Céligny P, Roy P, Colombat P, et al. Follicular lymphoma international prognostic index. Blood 2004;104(5):1258-1265 
6 Brice P, Bastion Y, Lepage E, et al. Comparison in low-tumor-burden follicular lymphomas between an initial no-treatment policy, prednimustine, or interferon alfa: a randomized study from the Groupe d'Etude des Lymphomes Folliculaires. Groupe d'Etude des Lymphomes de l'Adulte. J Clin Oncol 1997; 15(3):1110-1117

7 Ardeshna KM, Smith P, Norton A, et al. British National Lymphoma Investigation. Long-term effect of a watch and wait policy versus immediate systemic treatment for asymptomatic advanced-stage non-Hodgkin lymphoma: a randomised controlled trial. Lancet 2003;362(9383) :516-522

8 Wahlin BE, Yri OE, Kimby E, et al. Clinical significance of the WHO grades of follicular lymphoma in a population-based cohort of 505 patients with long follow-up times. Br J Haematol 2012;156(2):225-233

9 Gangatharan SA, Maganti M, Kuruvilla JG, et al. Clinical characteristics and early treatment outcomes of follicular lymphoma in young adults. Br J Haematol 2015;170(3):384-390

10 Conconi A, Lobetti-Bodoni C, Montoto S, et al. Life expectancy of young adults with follicular lymphoma. Ann Oncol 2015; 26(11):2317-2322

11 Casulo C, Day B, Dawson KL, et al. Disease characteristics, treatment patterns, and outcomes of follicular lymphoma in patients 40 years of age and younger: an analysis from the National Lymphocare Studyt. Ann Oncol 2015;26(11): 2311-2317
12 Summerfield GP, Wood KM, Taylor PR, White JM, Mounter PJ, Proctor SJ. Survival in young patients (less than 40 years) with follicular lymphoma: a population based study by the Scotland and Newcastle Lymphoma Group. Leuk Lymphoma 2004;45(6): 1149-1157

13 Casulo C, Byrtek M, Dawson KL, et al. Early relapse of follicular lymphoma after rituximab plus cyclophosphamide, doxorubicin, vincristine, and prednisone defines patients at high risk for death: an analysis from the National LymphoCare Study. J Clin Oncol 2015;33(23):2516-2522

14 Maurer MJ, Habermann TM, Shi Q, et al. Progression-free survival at 24 months (PFS24) and subsequent outcome for patients with diffuse large B-cell lymphoma (DLBCL) enrolled on randomized clinical trials. Ann Oncol 2018;29(8):1822-1827

15 Gogia A, Raina V, Kumar L, Sharma A, Sharma MCh, Mallick SR. Follicular lymphoma: an institutional analysis. Asian Pac J Cancer Prev 2017;18(3):681-685

16 Gupta AA, Edelstein K, Albert-Green A, D’Agostino N. Assessing information and service needs of young adults with cancer at a single institution: the importance of information on cancer diagnosis, fertility preservation, diet, and exercise. Support Care Cancer 2013;21(9):2477-2484

17 de Juan VC, Provencio M, Codina JG, Abreu DR, Rueda A, Arroyo RG, et al.916P- Survival in young adults diagnosed with follicular lymphoma in a national registry from the Spanish Lymphoma Oncology Group. Ann Oncol. 2016 Oct 1;27:vi316 